\title{
Influenza in the US Military: An Overview
}

\section{Abstract}

Circulation of influenza strains in the military has been responsible for clusters of illness among military personnel in the United States and remote areas where they operate, although not usually associated with a high degree of morbidity [1,2]. During the latest 5-year period (2007-2012) for which there is published data by the AFHSC, influenza was found to be responsible for as many as 7,000 to 25,000 cases per week in the MHS, of which 3,000 to 16,000 (40 to 65 percent) involved military personnel [3]. Since 2009, pH1N1 has continued to circulate worldwide; [4] it resurged in the US from November 2013 through February 2014, causing an increase in laboratory-confirmed influenza associated hospitalizations in all age groups [5].

Since summer-fall of 2014, drifted H3N2 viruses have begun to predominate causing an increase in laboratory-confirmed influenza associated hospitalizations among both US civilian and military personnel in the winter of 2014-2015 and through December $2015[1,6]$. These drifted H3N2 viruses have also been associated with increased mortality, especially among those older than 64 years [7-9].

Keywords: US military; Influenza; H1N1; H3N1; Armed forces

Received: February 08, 2016; Accepted: February 25, 2016; Published: March 05, 2016

\section{Clinical Aspects}

Seasonal influenza viruses (H1N1, H3N2 and B subtypes) have a very short incubation period (median, 2 days; range, 1 to 4 days), but may be longer (up to 8 to 9 days) in infections caused by other avian influenza viruses (AIVs) $[10,11]$. Viral shedding begins approximately 24 to 48 hours prior to symptom onset, peaks within 48 to 72 hours, and can continue for up to a week after symptom resolution, especially in non-immune individuals. Hospitalized adults may shed infectious virus for a week or longer after illness onset. Viremia rarely occurs in uncomplicated influenza except in cases of H5N1-infected patients, in whom detection of viral ribonucleic acid (RNA) in the blood is associated with a worsened prognosis.

Most persons with symptomatic influenza virus infection have uncomplicated illness with sudden onset of fever, cough, headaches, and malaise, all of which resolve over 3 to 5 days, although cough and fatigue may persist longer. Some adults may also experience diarrhea [12]. Although most persons with influenza virus infection will not develop critical illness, those who are pregnant $[13,14]$, obese $[13,15]$, suffer from chronic renal or liver disease, [16] or suffer from diseases of the circulatory
Jose L Sanchez ${ }^{1}$ and Michael J Cooper ${ }^{2}$

1 Armed Forces Health Surveillance Branch, Public Health Division, Defense Health Agency, 11800 Tech Road, Silver Spring, MD 20904, USA

2 Respiratory Program Initiatives, Global Emerging Infections System, Armed

Forces Health Surveillance Branch, Public Health Division, Defense Health Agency, 11800 Tech Road, Silver Spring, MD 20904, USA

Corresponding author: Jose L. Sanchez

” toti.sanchez@us.army.mill

MD, MPH, Armed Forces Health Surveillance Branch, 11800 Tech Road, Suite 218, Silver Spring, MD 20904, USA.

Citation: Sanchez JL, Cooper MJ. Influenza in the US Military: An Overview. J Infec Dis Treat. 2016, 2:1. 
is most likely an accurate reflection of influenza viruses' low virulence among military personnel because of the availability of real-time, systematic, standardized military data collection systems which appropriately and promptly evaluate influenza (or other pathogen)-related mortality.

\section{Diagnosis}

Influenza viruses can be readily isolated in tissue culture from nasal swab specimens, nasal aspirates, or combined nose and throat swab specimens $[20,21]$. As with adenoviruses, the time required to detect influenza viruses in cell culture can be shortened to one to two days by employing shell vial centrifugation culture (SVCC) systems followed by fluorescent antibody staining. Immunologic detection of influenza antigens in respiratory samples can be used for rapid diagnosis, and a large number of such rapid influenza diagnostic tests (RIDTs) are commercially available [22] They can provide results at bedside (within 15 minutes or less), thus, results are available in a clinically relevant time period to inform clinical decisions. Unfortunately, RIDT sensitivities have varied widely (10 to 80 percent) compared to viral culture or molecular detection and are largely dependent on the type of sample as well as on the patient's age and phase of illness [22]. RIDT sensitivity is lower in adults and elderly patients than in young children, whose nasal secretions may contain larger quantities of virus $[23,24]$. Similarly, sensitivity is likely to be higher early in the course of illness (within 48 to 72 hours of onset), when viral shedding is maximal. Thus, care should be exercised when utilizing RIDTs later in the course of illness as sensitivity can be low as viral shedding decreases [25]. RIDT specificity, on the other hand, has been very good ranging from $85 \%$ to $100 \%$, thus, they are good tests for "ruling-in" rather than "ruling out" influenza infection, especially when influenza activity is high in the community [22]. Two recent FDA-cleared assay systems that rely on instrument optics to determine an objective result, as opposed to a subjective read by the operator, hope to improve performance of RIDTs [26].

The gradual dissemination of nucleic acid amplification testing (NAAT), including real-time reverse transcriptase polymerase chain reaction ( $r$ RT-PCR), in clinical laboratories has shifted the focus of laboratory diagnosis of influenza from dependency on viral culture, which takes several days, to a highly specific (>99.9 percent) and sensitive (86 to 100 percent) diagnosis available within several hours [27]. Sample processing automation, combined with user-friendly platforms for NAAT testing and information management systems, facilitates high-throughput molecular diagnostics for the detection of viral nucleic acids, including influenza A, from a variety of respiratory tract samples. Molecular assays can be used in conjunction with other diagnostic assays, and with clinical and epidemiologic information, to assist in patient management and treatment [28].

Rapid detection platforms which are US Food and Drug Administration (FDA)-cleared, such as those consisting of multiplex PCR tests for influenza, also allow the detection of other respiratory agents, either as single viruses or as copathogens [29-34]. Among adult patients with ARI in one study using this type of testing in the United States in 2012-2013, 5\% to $8 \%$ were found to sustain viral coinfections, to include influenza,
HCoVs, RSV and HRV [35]. One influenza typing kit based on the RT-PCR electrospray ionization mass spectrometry (PCRESI-MS) platforms allows detection of all 16 hemagglutinin and 9 neuraminidase subtypes, [36] as well as detection of drift of specific genes over time [36-39]. Because of its ability to detect newly emerging recombinant, drifted, or shifted influenza viruses, the PCR-ESI-MS typing analysis can be useful in detecting newly emerging influenza strains [40]. New PCR-based point-of-care tests have been developed and are more sensitive ( $>90$ percent) than older RIDTs and are in wide use in the military [1,41-43].

\section{Treatment}

The only two classes of FDA-approved antivirals are available for early treatment and chemoprophylaxis of influenza infection are the $\mathrm{M} 2$ inhibitors (oral amantadine and rimantadine) and the neuraminidase inhibitors (NIs; oral oseltamivir, inhaled zanamivir and oral or inhaled laninamivir, not FDA-approved) [4447]. The ion channel protein present within the viral envelope (denominated $\mathrm{M} 2$ protein) is the target of the $\mathrm{M} 2$ inhibitors. By comparison, the NIs target the viral neuraminidase which acts on surface glycoproteins of the virus [48] The M2 inhibitors are active, in vitro and in vivo, against all strains of influenza $A$ virus; however, they are not active against influenza B viruses, and antiviral resistance has increased since the emergence of the $\mathrm{pH} 1 \mathrm{~N} 1$ strain in $2009-2010$. Therefore, they are not presently recommended for use in the United States [44].

In placebo-controlled randomized clinical trials (RCTs) oseltamivir has been found to be effective in reducing duration of influenza symptoms by 21 percent (from 123 hours down to 98 hours) as well as the risk of hospitalization by 65 percent ( 0.6 percent compared to 1.7 percent in placebo recipients) among adults in a recently published meta-analysis [49]. In addition to recommending influenza vaccine for preventing influenza, the CDC and the FDA recommend use of NIs for the treatment of influenza [44,50]. There are also a number of published observational studies providing data that support the efficacy of NIs (oral oseltamivir, inhaled zanamivir) for uncomplicated influenza, reducing fever and illness duration by approximately one day [51,52]. NIs have also been found to be effective in reducing mortality in patients hospitalized with $\mathrm{pH} 1 \mathrm{~N} 1$ virus infection including, but not limited to, pregnant women $[50,53]$.

Treatment should be started within 48 hours of symptom onset and administered for at least 5 days in uncomplicated cases. Increased duration for up to 10 days, or higher dose (e.g., $150 \mathrm{mg}$ twice daily in adults with normal renal function) may be necessary in the case of critically ill patients with respiratory failure or among immunocompromised patients in whom prolonged viral replication may occur in the lower respiratory tract $[17,18,44,52]$. Oral oseltamivir treatment begun more than 2 days after illness onset may also be of some benefit $[54,55]$. Fortunately, resistance is low to-date (less than 1-2 percent of isolates) among the prevailing seasonal viruses in the US and worldwide $[8,56]$.

The US military recommends treatment only for people hospitalized with confirmed, probable or suspected disease; treatment should be implemented as soon as the clinician suspects infection and should not await laboratory confirmation 
[57] The DoD also stockpiles oseltamivir and zanamivir as a contingency component of its pandemic preparedness plans (AFHSB, unpublished data). In patients with severe disease (such as pneumonia or ARDS), empirical broad-spectrum antimicrobial therapy should also be initiated to cover bacterial coinfections, with appropriate de-escalation of antimicrobials when lower respiratory bacterial cultures return with definitive results [19].

\section{Chemoprophylaxis}

Chemoprophylaxis has also been shown to be beneficial if given for at least 7 days post-exposure. Systematic reviews have found NIs, but not M2 agents, to provide some degree of protection as chemoprophylactic agents [58] Oseltamivir (in a dose of $75 \mathrm{mg}$ daily) and zanamivir (in a dose of $10 \mathrm{mg}$ daily), but not amantadine, have demonstrated to be efficacious both as seasonal and as post-exposure chemoprophylaxis of influenza in households (efficacy approximately 67 to 89 percent) [59]. However, they have not been shown to prevent community-wide transmission of influenza $[59,60]$.

In a unique study in the Singaporean military at the height of a pH1N1 epidemic in June 2009, the implementation of "ring chemoprophylaxis" (defined as geographically targeted containment by use of oseltamivir) of co-workers and sameunit members was elegantly demonstrated to be effective, in conjunction with prompt identification and isolation of infected personnel, in a restricted entry training setting. Inhaled laninamivir has also been shown to reduce secondary illness rates among household contacts (78\% efficacy) in a RCT and may represent a third option for chemoprophylaxis [61].

At the present time, US military health officials do not routinely recommend "mass" or "targeted" outbreak chemoprophylaxis with NIs. However, the potential use of oseltamivir chemoprophylaxis can and should be considered by military health officials, especially if there are operational considerations which justify its use (such as circulation of a highly virulent strain, among high-risk patients during outbreaks in confined facilities or homes, among unvaccinated health care providers (HCP), in a perceived or real compromise of the military mission, or during an overwhelming epidemic) [44].

\section{Vaccine Effectiveness Monitoring}

Continued surveillance and determination of influenza vaccine effectiveness (VE) has ongoing in the US military for many years. In collaboration with the US Centers for Disease Control and Prevention (CDC) and the FDA, the US military estimates midyear and year-end influenza VE and these are provided at the time of the FDA's Vaccine and Related Biologic Products Advisory Committee (VRBPAC) meetings. These analyses examine VE by type of vaccine (e.g., live attenuated vaccine or inactivated vaccine), status (military members versus non-military) and age strata each year in order to track VE in these groups. Methodological and immunological issues regarding estimates of VE in US military members have surfaced and have stimulated ongoing research regarding the potential impact of a frequent vaccination (i.e., multiple vaccinations for influenza in succession over multiple years) on antigenic response to current and future vacinations. In addition, questions regarding the possiblility of waning protection within a given influenza season suggests that later vaccinations (vaccinations closer to the peak) or multiple vaccinations within the influenza season might protect against infection more effectively. In general, it has been theorized that US military members might be different in important ways from civilian populations regarding VE and its estimation; therefore, military and dependent populations should continue to be be monitored specifically. Continued assessment of influenza VE in the US military is essential in order to continue to better inform vaccination policy decisions [62].

\section{Vaccine Develoment and Policy}

The US military requires influenza vaccination of military recruits as well as of all personnel on active duty status on a yearly basis [1]. The goal is to exceed 90-percent immunization of all military personnel by mid-December of each year; however, delays in receipt of vaccine and other logistic and access issues are taken into consideration, and all organizations are encouraged to continue their efforts to immunize throughout the influenza season [63]. Additionally, the US military's mandatory influenza vaccination policy, which applies to all of its $\mathrm{HCP}$, is based on the premise that vaccination is an important tool for enhancing patient safety and quality of care as well as a means of protecting patients and staff members $[64,65]$. Compliance among military HCP for 2012-2013 and 2013-2014 has been excellent with vaccination rates exceeding $95 \%$ each year [66]. By comparison, compliance rates among civilian HCP in the United States have not exceeded $75 \%$ [67].

The US military has played a key role in the development, deployment, and management of influenza vaccines for the entire nation. The US military led their development in the late 1930s when Dr's Jonas Salk and Thomas Francis developed the first inactivated vaccines which were used to protect US military personnel during World War II [68]. The US military was also the first institution which established a universal influenza vaccination policy which dates back to the early 1940s, many decades before widespread immunization of healthy young people was recommended by the $C D C$ and other international health officials $[1,69]$. For many years, the CDC recommended influenza immunization only for aged and infirmed people, while the US military was immunizing the entire force. Lastly, because of the important need for their protection, US military health officials have been an active participant in the annual vaccine strain selection activities led by the FDA which, in the past, sought a military member to be part of the FDA's VRBPAC.

Even though a large proportion of US military personnel are immunized with current influenza vaccines, influenza viruses continue to affect them; $[1,70]$ this is most likely multifactorial in nature. First, humoral-mediated immunity is transitory requiring annual immunizations [71]. This is most likely explained by distinct patterns of B-cell activation and priming resulting in lower cross-protection against heterovariant and heterosubtypic influenza strains [72]. Second, subtypes contained in annual vaccine formulations often do not match prevailing circulating 
subtypes, thus, vaccine-derived immunity is non-efficacious in many cases [73] Third, even under the best of circumstances, vaccine efficacy among healthy adults is no higher than 60 to 80 percent for inactivated vaccines and much lower for live attenuated formulations, leaving many vaccinees susceptible to infection [71,74]. Lastly, military personnel often travel or are deployed to areas of the world where influenza virus subtypes differ from those subtypes included in the US-based vaccines.

\section{Acknowledgements}

The views expressed herein are those of the authors and do not reflect the official policy or position of the Department of the Army, Department of Defense, or the US Government. Dr. Sanchez is a US government employee. This work was prepared as part of his official duties and, as such, there is no copyright to be transferred. 


\section{References}

1 Sanchez JL, Cooper MJ, Myers CA, Cummings JF, Vest KG, et al. (2015) Respiratory Infections in the U.S. Military: Recent Experience and Control. Clin Microbiol Rev 28: 743-800.

2 Parms TA, Zorich SC, Kramer KP (2015) Influenza A(H3N2) outbreak at Transit Center at Manas, Kyrgyzstan, 2014. MSMR 22: 7-10.

3 (2012) Update: pneumonia-influenza and severe acute respiratory illnesses, active component, U.S. Armed Forces, July 2000-June 2012. MSMR 19: 11-13.

4 World Health Organization (2015) Global Influenza Programme, FluNet Summary.

5 Epperson S, Blanton L, Kniss K, Mustaquim D, Steffens C, et al. (2014) Influenza activity - United States, 2013-14 season and composition of the 2014-15 influenza vaccines. MMWR Morb Mortal Wkly Rep 63: $483-490$.

6 US Air Force School of Aerospace Medicine (2016) DoD Global, Laboratory-based, Influenza Surveillance Program Report, 13-26 December 2015.

7 Centers for Disease Control and Prevention (2014) Health Advisory Regarding the Potential for Circulation of Drifted Influenza A (H3N2) Viruses. CDCHAN-00374, 3 December 2014.

8 D'Mello T, Brammer L, Blanton L, Kniss K, Smith S, et al. (2015) Update: Influenza activity--United States, September 28, 2014-February 21, 2015. MMWR Morb Mortal Wkly Rep 64: 206-212.

9 Rolfes M, Blanton L, Brammer L, Smith S, Mustaquim D, et al. (2014) Update: influenza activity - United States, September 28- December 6, 2014. MMWR Morb Mortal Wkly Rep 63: 1189-1194.

10 Lessler J, Reich NG, Brookmeyer R, Perl TM, Nelson KE, et al. (2009) Incubation periods of acute respiratory viral infections: a systematic review. Lancet Infect Dis 9: 291-300.

11 Hayden FG, de Jong MD (2013) Human influenza: Pathogenesis, clinical features, and management. Textbook of Influenza. 2nd edn. In: Webster RG MA, Braciale TJ, Lamb RA (eds) West Sussex, UK: John Wiley \& Sons pp: 373-391.

12 Uyeki TM (2014) Preventing and controlling influenza with available interventions. N Engl J Med 370: 789-791.

13 Karlsson EA, Marcelin G, Webby RJ, Schultz-Cherry S (2012) Review on the impact of pregnancy and obesity on influenza virus infection. Influenza Other Respir Viruses 6: 449-460.

14 Siston AM, Rasmussen SA, Honein MA, Fry AM, Seib K, et al. (2010) Pandemic 2009 influenza $A(H 1 N 1)$ virus illness among pregnant women in the United States. JAMA 303: 1517-1525.

15 Zhou Y, Cowling BJ, Wu P, Chan WM, Lee SY, et al. (2015) Adiposity and influenza-associated respiratory mortality: a cohort study. Clin Infect Dis 60: e49-57.

16 Van Kerkhove MD, Cooper MJ, Cost AA, Sanchez JL, Riley S (2015) Risk factors for severe outcomes among members of the United States military hospitalized with pneumonia and influenza, 20002012. Vaccine 33: 6970-6976.

17 Writing Committee of the WHO Consultation on Clinical Aspects of Pandemic (H1N1) (2009) Clinical aspects of pandemic 2009 influenza A (H1N1) virus infection. N Engl J Med 362: 1708-1719.

18 Napolitano LM, Angus DC, Uyeki TM (2014) Critically ill patients with influenza $A(H 1 N 1)$ pdm09 virus infection in 2014. JAMA 311: 1289-1290.
19 Lee FE-H, Treanor J (2010) Viral infections. Textbook of Respiratory Medicine (6th edn) In: Mason RJ, Broaddus VC, Martin TR, et al. (eds), Philadelphia, PA: Saunders Elsevier Inc. pp: 661-698.

20 Treanor JJ (2015) Influenza (Including Avian Influenza and Swine Influenza). Principles and Practice of Infectious Disease (8th edn) In: Bennett JE, Dolin R, Blaser MJ (eds), Philadelphia, PA: Churchill Livingstone Elsevier pp: 2000-2024.

21 Centers for Disease Control and Prevention (2013) Guidance for clinicians on the use of rapid influenza diagnostic tests.

22 Faix DJ, Sherman SS, Waterman SH (2009) Rapid-test sensitivity for novel swine-origin influenza A (H1N1) virus in humans. N Engl J Med 361: 728-729.

23 Hurt AC, Baas C, Deng YM, Roberts S, Kelso A, et al. (2009) Performance of influenza rapid point-of-care tests in the detection of swine lineage $A(\mathrm{H} 1 \mathrm{~N} 1)$ influenza viruses. Influenza Other Respir Viruses 3: 171-176.

24 Babin SM, Hsieh YH, Rothman RE, Gaydos CA (2011) A meta-analysis of point-of-care laboratory tests in the diagnosis of novel 2009 swine-lineage pandemic influenza A (H1N1). Diagn Microbiol Infect Dis 69: 410-418.

25 Leonardi GP, Wilson AM, Mitrache I, Zuretti AR (2015) Comparison of the Sofia and Veritor Direct Antigen Detection Assay Systems to Identify Influenza Viruses from Patient Nasopharyngeal Specimens. J Clin Microbiol 53: 1345-1347.

26 Zambon M (2013) Influenza surveillance and laboratory diagnosis. Textbook of Influenza (2nd edn) In: Webster RG MA, Braciale TJ, Lamb RA (eds) West Sussex, UK: John Wiley \& Sons pp: 231-249.

27 Centers for Disease Control and Prevention (2015) Guidance for clinicians on the use of RT-PCR and other molecular assays for diagnosis of influenza virus infection.

28 BioFire Diagnostics Inc. (2014) BiofireFilmArray Respiratory Panel.

29 Caliendo AM (2011) Multiplex PCR and emerging technologies for the detection of respiratory pathogens. Clin Infect Dis 52 Suppl 4: S326-330.

30 Deyde VM, Sampath R, Garten RJ, Blair PJ, Myers CA, et al. (2010) Genomic signature-based identification of influenza A viruses using RT-PCR/electro-spray ionization mass spectrometry (ESI-MS) technology. PLoS One 5: e13293.

31 Dunn JJ, Miller MB (2014) Emerging respiratory viruses other than influenza. Clin Lab Med 34: 409-430.

32 Ginocchio CC (2011) Strengths and weaknesses of FDA-approved/ cleared diagnostic devices for the molecular detection of respiratory pathogens. Clin Infect Dis 52 Suppl 4: S312-325.

33 Peaper DR, Landry ML (2014) Rapid diagnosis of influenza: state of the art. Clin Lab Med 34: 365-385.

34 Zimmerman RK, Rinaldo CR, Nowalk MP, Balasubramani GK, et al. (2015) Viral infections in outpatients with medically attended acute respiratory illness during the 2012-2013 influenza season. BMC Infect Dis 15: 87.

35 Sampath R, Russell KL, Massire C, Eshoo MW, Harpin V, et al. (2007) Global surveillance of emerging Influenza virus genotypes by mass spectrometry. PLoS One 2: e489.

36 Deyde VM, Sampath R, Gubareva LV (2011) RT-PCR/electrospray ionization mass spectrometry approach in detection and characterization of influenza viruses. Expert Rev Mol Diagn 11: 41-52. 
37 Jeng K, Massire C, Zembower TR (2012) Monitoring seasonal influenza A evolution: rapid 2009 pandemic H1N1 surveillance with an reverse transcription-polymerase chain reaction/electro-spray ionization mass spectrometry assay. J Clin Virol 54: 332-336.

38 Tang YW, Lowery KS, Valsamakis A, Schaefer VC, Chappell JD, et al. (2013) Clinical accuracy of a PLEX-ID flu device for simultaneous detection and identification of influenza viruses $A$ and $B$. J Clin Microbiol 51: 40-45.

39 Metzgar D, Baynes D, Myers CA, Kammerer P, Unabia M, et al. (2010) Initial identification and characterization of an emerging zoonotic influenza virus prior to pandemic spread. J Clin Microbiol 48: 4228 4234.

40 DiMaio MA, Sahoo MK, Waggoner J, Pinsky BA (2012) Comparison of Xpert Flu rapid nucleic acid testing with rapid antigen testing for the diagnosis of influenza A and B. J Virol Methods 186: 137-140.

41 Food and Drug Administration (2015) FDA grants first CLIA waiver for nucleic acid-based flu diagnostic test.

42 Novak-Weekley SM, Marlowe EM, Poulter M, Dwyer D, Speers D, et al. (2012) Evaluation of the Cepheid Xpert Flu Assay for rapid identification and differentiation of influenza A, influenza A 2009 H1N1, and influenza B viruses. J Clin Microbiol 50: 1704-1710.

43 Centers for Disease Control and Prevention (2014) Influenza antiviral medications: summary for clinicians.

44 Fiore AE, Fry A, Shay D, Gubareva L, Bresee JS, et al. (2011) Antiviral agents for the treatment and chemoprophylaxis of influenza --recommendations of the Advisory Committee on Immunization Practices (ACIP). MMWR Recomm Rep 60: 1-24.

45 United States Food and Drug Administration (2014) FDA approves Rapivab to treat flu infection.

46 Ison MG (2013) Clinical use of approved influenza antivirals: therapy and prophylaxis. Influenza Other Respir Viruses 7 Suppl 1: 7-13.

47 Zumla A, Memish ZA, Maeurer M, Bates M, Mwaba P, et al. (2014) Emerging novel and antimicrobial-resistant respiratory tract infections: new drug development and therapeutic options. Lancet Infect Dis 14: 1136-1149.

48 Dobson J, Whitley RJ, Pocock S, Monto AS (2015) Oseltamivir treatment for influenza in adults: a meta-analysis of randomised controlled trials. Lancet 385: 1729-1737.

49 United States Food and Drug Administration (2015) Influenza (Flu) antiviral drugs and related information.

50 Harper SA, Bradley JS, Englund JA (2009) Seasonal influenza in adults and children--diagnosis, treatment, chemoprophylaxis, and institutional outbreak management: clinical practice guidelines of the Infectious Diseases Society of America. Clin Infect Dis 48: 10031032.

51 Santesso N, Hsu J, Mustafa R, Brozek J, Chen YL, et al. (2013) Antivirals for influenza: a summary of a systematic review and meta-analysis of observational studies. Influenza Other Respir Viruses 7 Suppl 2: 76-81.

52 Muthuri SG, Venkatesan S, Myles PR, Leonardi-Bee J, A Khuwaitir TS, et al. (2014) Effectiveness of neuraminidase inhibitors in reducing mortality in patients admitted to hospital with influenza $A$ H1N1pdm09 virus infection: a meta-analysis of individual participant data. Lancet Respir Med 2: 395-404.

53 Muthuri SG, Myles PR, Venkatesan S, Leonardi-Bee J, NguyenVan-Tam JS (2013) Impact of neuraminidase inhibitor treatment on outcomes of public health importance during the 2009-2010 influenza $A(H 1 N 1)$ pandemic: a systematic review and meta-analysis in hospitalized patients. J Infect Dis 207: 553-563.

54 Rasmussen SA, Jamieson DJ (2014) 2009 H1N1 influenza and pregnancy--5 years later. N Engl J Med 371: 1373-1375.

55 Meijer A, Rebelo-de-Andrade H, Correia V, Besselaar T, DragerDayal R, et al. (2014) Global update on the susceptibility of human influenza viruses to neuraminidase inhibitors, 2012-2013. Antiviral Res 110: 31-41.

56 Assistant Secretary of Defense (Health Affairs) (2007) Policy for Release of Department of Defense Antiviral Stockpile during an Influenza Pandemic (HA Policy 07-015).

57 Jackson RJ, Cooper KL, Tappenden P, Rees A, Simpson EL, et al. (2011) Oseltamivir, zanamivir and amantadine in the prevention of influenza: a systematic review. J Infect 62: 14-25.

58 Hayden FG, Belshe R, Villanueva C, Lanno R, Hughes C, et al. (2004) Management of influenza in households: a prospective, randomized comparison of oseltamivir treatment with or without postexposure prophylaxis. J Infect Dis 189: 440-449.

59 Okoli GN, Otete HE, Beck CR, Nguyen-Van-Tam JS (2014) Use of neuraminidase inhibitors for rapid containment of influenza: a systematic review and meta-analysis of individual and household transmission studies. PLoS One 9: e113633.

60 Kashiwagi S, Watanabe A, Ikematsu H, Awamura S, Okamoto T, et al. (2013) Laninamivir octanoate for post-exposure prophylaxis of influenza in household contacts: a randomized double blind placebo controlled trial. J Infect Chemother 19: 740-749.

61 Eick-Cost AA, Tastad KJ, Guerrero AC (2012) Effectiveness of seasonal influenza vaccines against influenza-associated illnesses among US military personnel in 2010-11: a case-control approach. PLoS One 7: e41435.

62 Immunization Healthcare Branch (2004) Public Health Division - Defense Health Agency. Immunization Goal for the 2013-2014 Influenza Season.

63 Immunization Healthcare Branch (2008) Public Health Division Defense Health Agency, Vaccine Healthcare Centers Network. Policy for Mandatory Seasonal Influenza Immunization for Civilian Health Care Personnel Who Provide Direct Patient Care in Department of Defense Military Treatment Facilities.

64 Immunization Healthcare Branch (2005) Public Health Division - Defense Health Agency, Vaccine Healthcare Centers Network. Mandatory Influenza Vaccination for all DoD Health Care Workers.

65 Armed Forces Health Surveillance Center (AFHSC) (2014) Surveillance snapshot: Influenza immunization among U.S. Armed Forces healthcare workers. MSMR 21: 20.

66 Black CL, Yue X, Ball SW, Donahue SM, Izrael D, et al. (2014) Influenza vaccination coverage among health care personnel--United States, 2013-14 influenza season. MMWR Morb Mortal Wkly Rep 63: 805-811.

67 Hilleman MR (2000) Vaccines in historic evolution and perspective: a narrative of vaccine discoveries. Vaccine 18: 1436-1447.

68 Grabenstein JD, Pittman PR, Greenwood JT, Engler RJ (2006) Immunization to protect the US Armed Forces: heritage, current practice, and prospects. Epidemiol Rev 28: 3-26.

69 Jeffery DD, Cohen M, Brooks A, Linton A, Gromadzki R, et al. (2013) Impact of the 2009 influenza (H1N1) pandemic on the United States military health care system. Mil Med 178: 653-658. 
70 Monto AS, Ohmit SE (2009) Seasonal influenza vaccines: evolutions and future trends. Expert Rev Vaccines 8: 383-389.

71 He XS, Holmes TH, Sanyal M (2014) Distinct patterns of B-cell activation and priming by natural influenza virus infection versus inactivated influenza vaccination. J Infect Dis 211: 1051-1059.

72 Tricco AC, Chit A, Soobiah C, Hallett D, Meier G, et al. (2013) Comparing influenza vaccine efficacy against mismatched and matched strains: a systematic review and meta-analysis. BMC Med 11: 153.

73 Nichol KL (2008) Efficacy and effectiveness of influenza vaccination. Vaccine 26 Suppl 4: D17-22.

74 Osterholm MT, Kelley NS, Sommer A, Belongia EA (2012) Efficacy and effectiveness of influenza vaccines: a systematic review and metaanalysis. Lancet Infect Dis 12: 36-44. 